\title{
Upcoming market catalysts in Q4 2013
}

Exciting developments for orphan drugs and therapies with breakthrough designation are expected in the fourth quarter of 2013. Notable market catalysts include a US Food and Drug Administration (FDA) advisory panel meeting for tasimelteon (developed by Vanda Pharmaceuticals and Bristol-Myers Squibb) for non-24-hour sleep-wake disorder (Non-24) in the totally blind and an FDA decision on the approval of obinutuzumab (developed by Roche) for patients with previously untreated chronic lymphocytic leukaemia (CLL). Additionally, top-line Phase III data are anticipated from drisapersen (developed by GlaxoSmithKline and Prosensa) in males with Duchenne muscular dystrophy (DMD).

Tasimelteon, a dual melatonin receptor agonist, was granted orphan status by the FDA and the European Medicines Agency (EMA) for Non-24 disorder in the totally blind. The disorder is characterized by an inability to regulate circadian rhythm and affects $\sim 50 \%$ of totally blind individuals because their brains fail to receive external lighting cues. Vanda conducted two pivotal studies, known as SET and RESET, in which tasimelteon demonstrated significant improvements on the primary end point of maintenance of circadian rhythm entrainment. The SET study also showed significance on its co-primary end point of clinical response, which was defined as entrainment and a score of $\geq 3$ in the Non-24 Clinical Response Scale. As there are no approved drugs for Non-24 disorder, end points for this indication are undefined. Consequently, the SET study initially sought to evaluate average total night time sleep as the primary end point; however, Vanda revised this in June 2012 to the new entrainment end point and again in November 2012 to include the clinical response co-primary end point. An FDA advisory committee meeting is tentatively scheduled for 14 November 2013. There is ongoing debate about whether entrainment or total sleep time is more important, and it will be interesting to see how panel members view the end

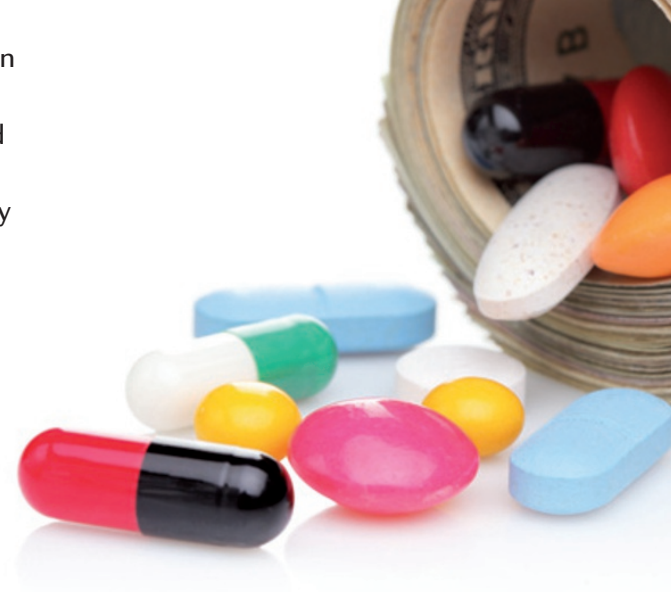

points and if they find the entrainment data compelling enough to warrant approval.

Breakthrough therapy designation was introduced in the United States with the Food and Drug Administration Safety and Innovation Act in July 2012: in order to be considered, drugs are required to demonstrate preliminary efficacy on at least one clinical end point compared with current therapies. Obinutuzumab received this designation in May 2013 as a first-line treatment for patients with CLL. Results from the first stage of the pivotal CLL11 trial were presented at the 2013 American Society of Clinical Oncology meeting. Obinutuzumab plus chlorambucil outperformed both chlorambucil monotherapy (median progression-free survival (PFS): 23 months versus 10.9 months; overall response rate (ORR): $75.5 \%$ versus $30.2 \%$ ) and rituximab plus chlorambucil (median PFS: 23 months versus 15.7 months; ORR: $75.5 \%$ versus $65.9 \%$ ). Subsequently, data from the second stage of the trial comparing obinutuzumab head-to-head against rituximab, which were both in combination with chlorambucil, demonstrated a significant improvement on PFS, thus validating results from the first stage of the trial. Detailed results are expected to be presented at the American Society of Hematology meeting from the 7 to 10 December 2013. Given the overwhelmingly positive results from CLL11, obinutuzumab is poised to become the first

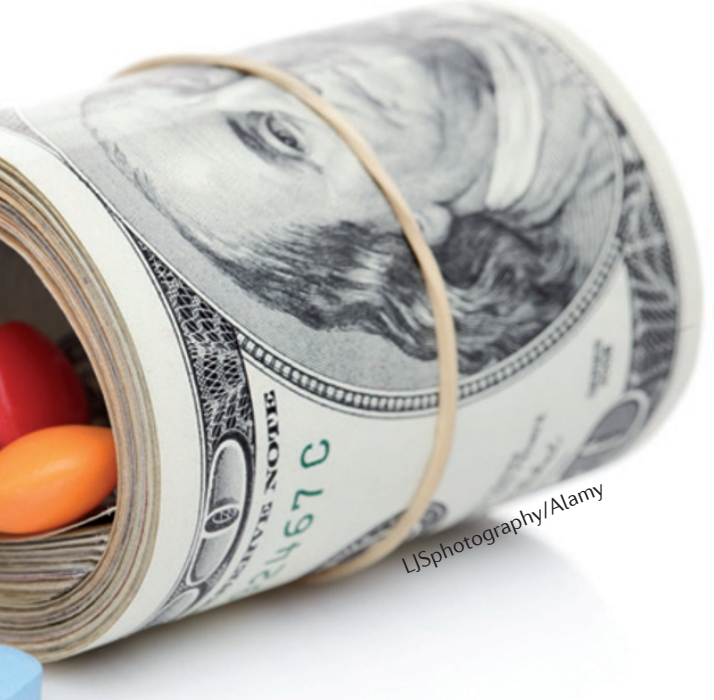

approved breakthrough therapy, as it has a Prescription Drug User Fee Act (PDUFA) date of 20 December 2013.

Drisapersen has received both orphan and breakthrough designations for DMD, a disease in which mutations in dystrophin (an important structural component in muscle tissue) lead to muscle degeneration and weakness. Drisapersen is an antisense drug that induces skipping of a common mutation in the dystrophin gene, allowing the formation of a functional protein. The ongoing Phase III DMD114044 study has enrolled 186 ambulant boys and is comparing drisapersen to placebo. Top-line data for the primary end point the change in 6-minute walk distance - are expected this quarter. In February 2013, the companies disclosed that four patients enrolled in ongoing Phase II and III studies were hospitalized due to thrombocytopenia and severe proteinuria, but have recovered and are continuing per protocol. A previous 53-patient Phase II study (known as DMD114117) highlighted proteinuria as a common adverse event, occurring in 11-18\% of patients compared with $11 \%$ for placebo, and no reports of thrombocytopenia. Owing to a lack of treatment options, these safety findings may not preclude approval, but we remain cautious and look for further details in the Phase III study.

Stacey Fong is a BioMedTracker Principal Research Analyst at Sagient Research Systems, 3655 Nobel Drive, San Diego, California 92122, USA. e-mail:sfong@sagientresearch.com

The author declares no competing financial interests. 